\title{
Self-similar space-filling packings in three dimensions
}

\author{
Reza Mahmoodi Baram, Hans J. Herrmann \\ August 30, 2018 \\ Institute for Computational Physics, University of Stuttgart, \\ Pfaffenwaldring 27, 70569 Stuttgart, Germany \\ reza@ica1.uni-stuttgart.de,hans@ica1.uni-stuttgart.de
}

\begin{abstract}
We develop an algorithm to construct new self-similar space-filling packings of spheres. Each topologically different configuration is characterized by its own fractal dimension. We also find the first bicromatic packing known up to now.
\end{abstract}

\section{INTRODUCTION}

Apart from their geometrical beauty, self similar space filling packings of spheres are used as models for ideally dense granular packings. For example, although we cannot expect to reproduce experimentally the precise positions of their spheres, the size distribution of such packings are good candidates to be used in making high performance concrete, if a grain of sand can be approximated as a spherical particle.

The well-known Apollonian packing of circles is a two-dimensional example of such packings (see Fig.1). In two dimensions, Herrmann et al $[1,2]$ have developed an algorithm to produce a variety of different packings of circles among which the simple Apollonian packing is a special case.

Only one space filling packing in three dimension had been constructed and studied before (see for example Ref.[3,4]). Peikert et al[4] use a quite efficient method called inversion algorithm to produce this three dimensional Apollonian packing. The inversion algorithm is based on a simple conformal transformation, namely, inversion with respect to spheres[5]. 


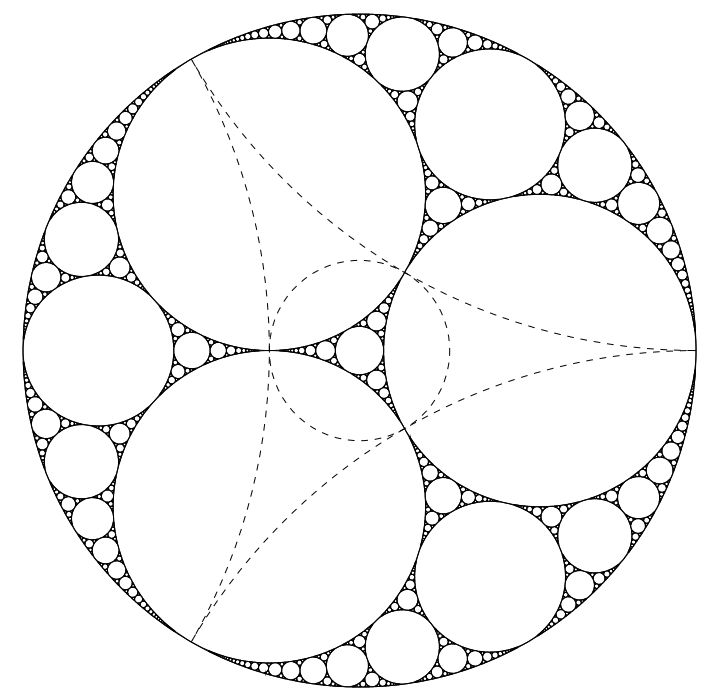

Figure 1: Apollonian packing of circles. The dashed circles are the inversion circles.

In this work, we show in detail how the inversion algorithm can be adapted to make four more topologically different packings of spheres, including a packing with the important property of having only two classes of spheres such that no spheres from the same class touch each other. We refer to this packing as the bichromatic packing which will be studied in more detail in a separate paper[6]. We also calculate the fractal dimensions of new packings. Different fractal dimensions imply the topological difference of structures of the packings.

In Sec.2 we explain in detail the inversion algorithm and two ways of its generalization in producing packings of circles. In Sec.3 we go over to three dimensions and discuss all possible packings of spheres which can be obtained using our method. Finally, in Sec.4 we calculate the fractal dimensions of the obtained packings and of two dimensional cuts of the bichromatic packings.

\section{PACKINGS in TWO DIMENSIONS}

Figure1 illustrates how the inversion algorithm can be employed to construct the classic Apollonian packing of circles within an enveloping circle of unity 


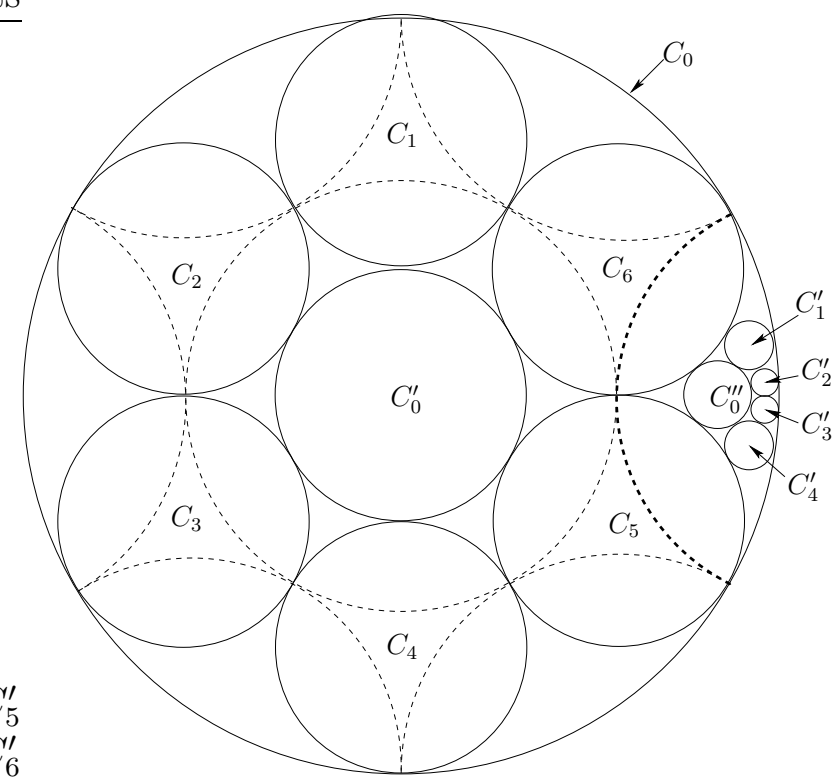

Figure 2: Initial circles on the six vertices of a hexagon.

radius. Initially three mutually touching circles are inscribed inside a circular space which is to be filled. Four inversion circles are set such that each of them is perpendicular to three of the four circles (three initial circles and the enveloping unit circle.) Beginning with this configuration, if all points outside an inversion circle are mapped inside, one new circle is generated, since the image of a circle perpendicular to the inversion circle falls on itself[5]. If the same is done for all the inversion circles, four new circles are generated inside the corresponding inversion circle. We call this the first generation. For next generations we simply continue by applying the inversions to the newly generated circles. As mentioned before, the inversion is made only from the outside to the inside of the inversion circles, otherwise the circles of previous generations would be generated again. In the limit of infinite generations we obtain the well-known Apollonian packing, in which the circular space is completely filled with circles of many sizes. Intuitively, there are two reasons why the inversion algorithm fills the space. On one hand, each of the inversion circles maps a larger more filled space into a smaller less filled space. On the other hand, every initially unoccupied space is inside at least 

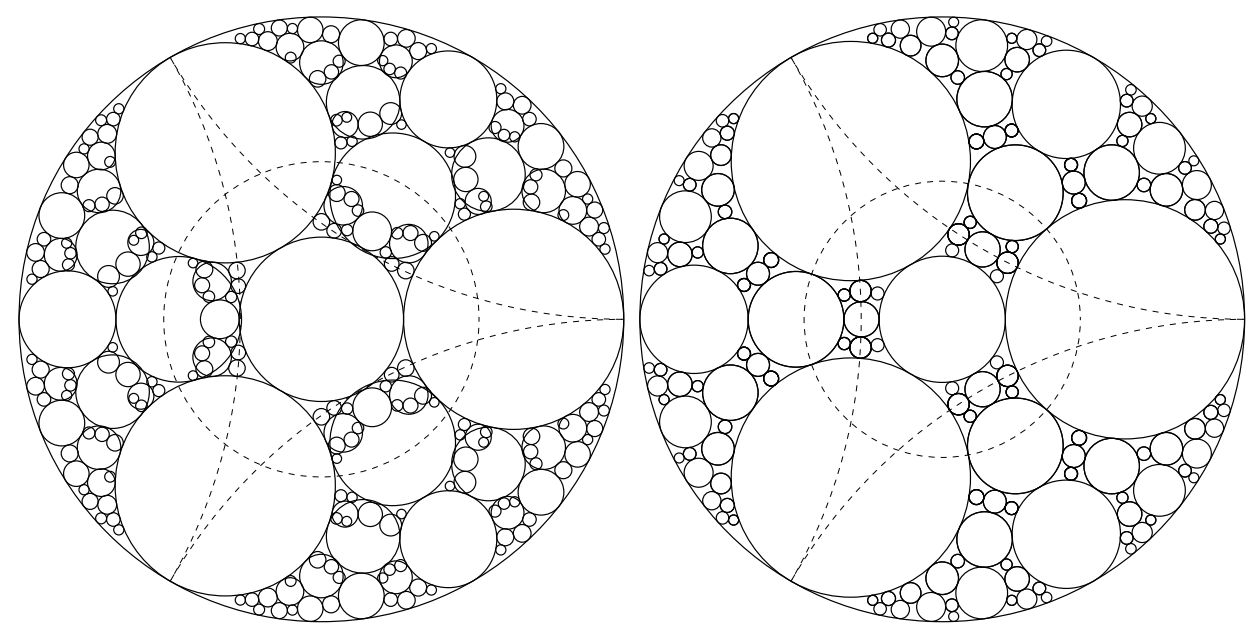

Figure 3: The generalization of the simple Apollonian packing to nontouching initial circles. If the angle between the inner and the outer inversion circles is chosen according to Eq.(1) a packing without overlapping is obtained (right image, corresponding to $l=0$ ), otherwise no physical packing is obtained (left image.)

one inversion circle.

The inversion algorithm in two dimensions can be generalized in two ways. One is to begin with initial circles on vertices of a regular polygon of $N$ sides inside the circular space instead of a triangle in the case of the simple Apollonian packing. Then, we will need $N+1$ inversion circles; $N$ inversion circles perpendicular to enveloping unit circle and to each pair of the initial circles which share the same side of the polygonal and one inversion circle in the center perpendicular to all initial circles (see Fig.2.) This generalization will inspire us also in generalizing the three dimensional inversion algorithm.

The second way is to begin with non-touching initial circles. We do this by reducing their sizes ${ }^{1}$. The outer inversion circles are same as before. But, the inner inversion circle should be larger in order to still be perpendicular to the initial circles. This, however, causes overlapping of inner inversion circles with outer ones. This might cause a problem, because the overlapping regions

\footnotetext{
${ }^{1}$ They will be still touching the border of the circular space, since otherwise the condition of having no initially unoccupied space outside all the inversion circles is not fulfilled
} 
correspond to two different larger regions which are not necessarily mapped exactly on top of each other. Fig.3 on the right shows the case when the size of the initial circles is chosen properly (as is explained in following) and on the left the case when size of the initial is arbitrarily chosen. As can be seen, in the later case no physical packing is obtained.

To handle this problem, we take a closer look at what happens in a sequence of alternating inversions of an arbitrary circle $C_{0}$ with respect to two overlapping inversion circles. This is shown in Fig. 4 on the left. Beginning with one of the inversion circles, we obtain an image for the circle $C_{0}$ inside the overlapping region, which is not necessarily the same as the one which is obtained beginning with the other inversion circle. However, for some certain values of $\alpha$, which is the angle between the tangents of the inversion circles in their intersection point, two images fall exactly on top of each other. To find these values, we transform the whole configuration shown on the left of Fig.4 by an inversion with respect to a circle centered around one of the intersecting points of two inversion circles. The result is shown on the right of the figure. The inversion circles become straight lines and act like mirrors. One can see that under the condition

$$
\alpha=\frac{\pi}{l+3}, \quad l=0,1,2,3 \cdots
$$

two images fall exactly on top of each other ${ }^{2}$.

Considering Eq.(1) in choosing the size of initial circles, we obtain an infinite number of different packings corresponding to the different values of $l \geq 0$ and $N \geq 3$. The only remaining problem is that each generated circle in the overlapping region is generated more than once, which can be easily solved by eliminating the repeated images. The packing with $l=0$ and $N=3$ is shown in Fig.3 on the right, together with the case which doesn't fulfill Eq.(1) on the left. The classic Apollonian packing of circles shown in the Fig. 1 corresponds to $l=\infty$ and $N=3$.

The packings we obtain here turn out to be the same as those constructed and named as first family of space-filling bearings by Herrmann et al [1] with $m=N-3$ and $n=l$ in their nomenclature. Fig.3 (on the right) is corresponding to $m=0, n=0$ as they classify it. All these packings

\footnotetext{
${ }^{2} \alpha=\frac{\pi}{2}$ corresponds to the case when the size of initial circles is zero and the circular space is completely filled by only one circle.
} 
are deterministic, self-similar fractal with a dimension which is different for different $n$ and $m$.

One may note that Eq.(1) should also hold in three dimensions when we deal with overlapping inversion spheres, since the cross section of any plane passing through the centers of the spheres gives the same situation as in two dimensions.

\section{GENERALIZATION to THREE DIMEN- SIONS}

As was mentioned before, Peikert et al used the inversion algorithm to very efficiently make the, so-called, three dimensional Apollonian packing of spheres within an enveloping sphere of radius one. In this section, we use this algorithm and its generalization discussed in the previous section to produce more such three dimensional self-similar space-filling packings in three dimensions.

Peikert et al begin with four initial spheres on the vertices of a tetrahedron inside a unit sphere which is to be filled. They placed five inversion spheres, four spheres perpendicular to the enveloping unit sphere and the three initial spheres belonging to each face of the tetrahedron, and one in the center perpendicular to all four initial spheres. However, the inversion spheres, even in this case which is the simplest three dimensional configuration, do overlap. We come back to this later in this section.

The fact that in two dimensions we can replace a triangle by other regular polygons as the basis for new configurations of initial circles suggests to do a similar extension in three dimensions. So, we examine the configurations based on the other Platonic Solids besides the tetrahedron as possible candidates. In this section, we will study all these cases and their resulting packings. An important restriction is, however, that the number of Platonic Solids is limited to five. Furthermore, as we will see in the following, the inversion spheres overlap in all configurations. Therefore we do not expect as many packings as in two dimensions. Actually, we will end up with only five packings.

We begin with mutually touching initial spheres on the vertices of a Platonic Solid inside the unit sphere. Inversion spheres are placed as follows; One in the center of the unit sphere perpendicular to all initial spheres, and 

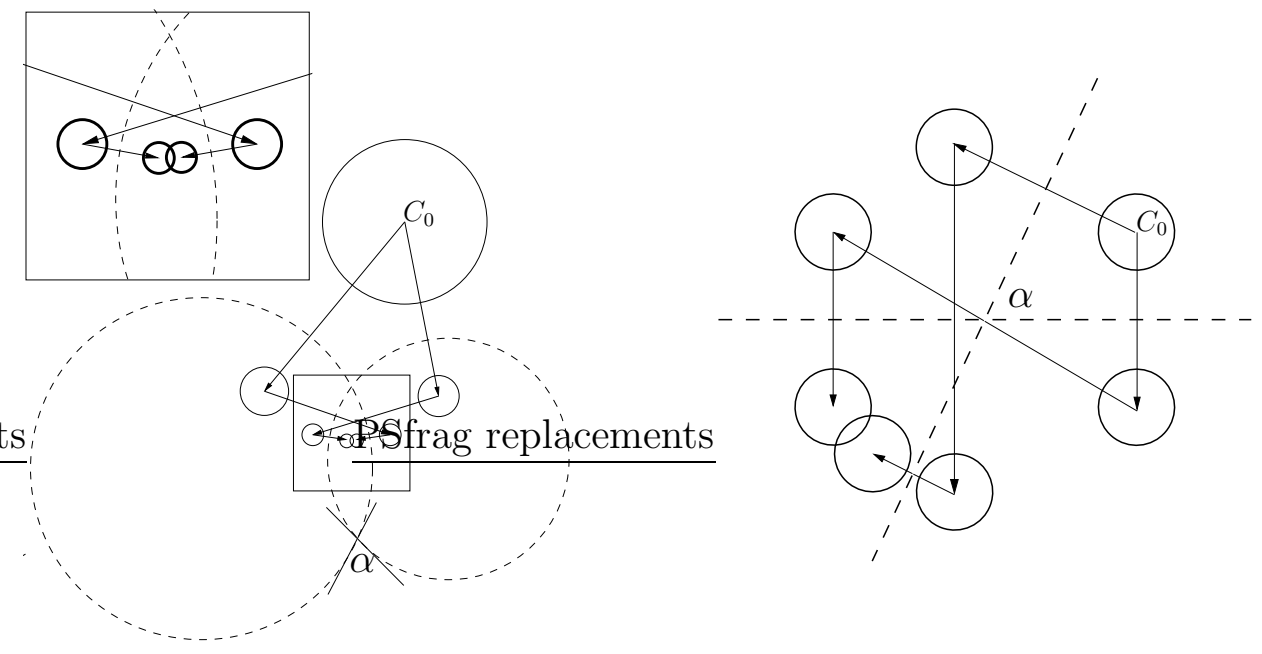

Figure 4: Iterative inversions of a circle $C_{0}$ with respect to two overlapping inversion circles (dashed lines). Two figures are equivalent (see the text).

one at each face of the Platonic Solid perpendicular to the enveloping unit sphere and the initial spheres which form that face of the Platonic Solid. Using this configuration of initial and inversion spheres, the process of filling the space is exactly the same as explained in the last section, that is, iteratively mapping the initial spheres into smaller and smaller unoccupied spaces. Figure 5 shows the packing based on the tetrahedron. The image on the left shows the packing after one generation and the one on the right shows the same packing including all spheres with radii larger than $2^{-7}$. The spheres are grouped into different classes (assigned by different colors) such that no spheres having the same color touch each other. For the tetrahedron-based packing there are five such classes of spheres, as can be seen in the figure.

As we discussed in the last section, in order that the iterative inversions lead to an allowed packing, the inversion spheres should either not overlap or, if they do, the angle $\alpha$ between them should follow Eq.(1). In this case 

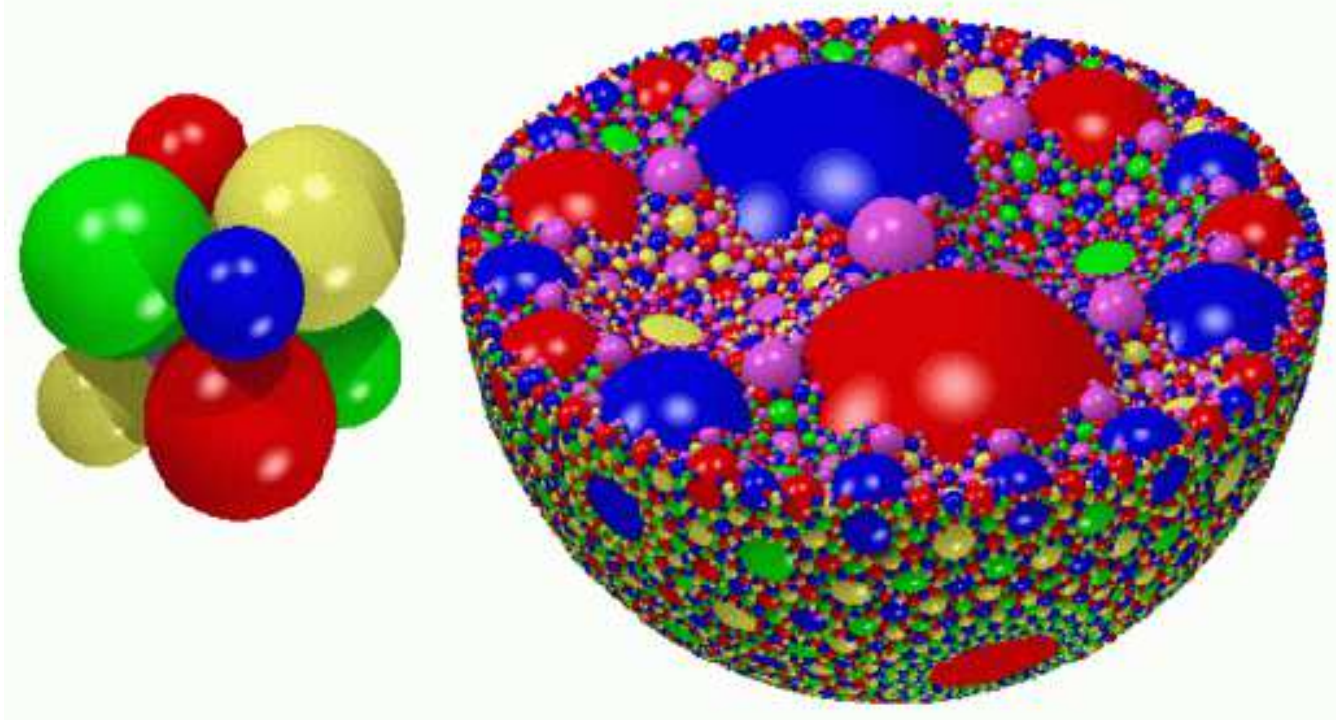

Figure 5: The tetrahedron-based packing.

$\alpha$ is the angle between the planes tangent to two inversion spheres at their intersetion points. We check the value of $\alpha$ for each Platonic Solid. To do this, we note that the centers of the inversion spheres are on the vertices of the conjugate Platonic Solid. As an example, Fig.6 shows the plane containing one of the faces of the Platonic Solid conjugate to the tetrahedron (which is also a tetrahedron). This plane is tangent to the unit sphere and to one of the initial spheres at their contact point. The circles are the cross section of the plane with the inversion spheres. One can easily see that the angle between two neigbouring inversion spheres is $\frac{\pi}{3}$, which happens to be equal to the angle corresponding to $l=0$ in Eq.(1). The corresponding angle between the inner and the outer inversion spheres can be also calculated and is $\frac{\pi}{3}$ too. Therefore an allowed configuration will be constructed. Because with this angle the overlapping of the invertion spheres will cause no problem but only multi-generation of spheres in overlapping region. As in last section, this problem is solved by eliminating the repeated spheres.

The first new packing is based on the octahedron, and in this case the angles between ovelapping inversion spheres again follow Eq.(1), having the 


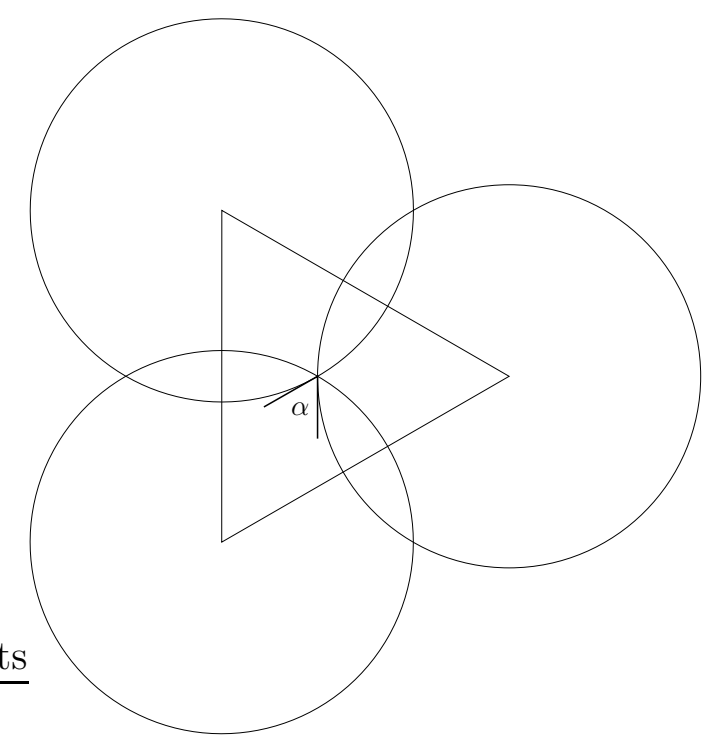

Figure 6: Overlapping of the inversion spheres in the case of tetrahedron. $\alpha$ equals $\frac{\pi}{3}$.

value $\frac{\pi}{4}$ (note that the conjugate of an octahedron is a cube whose faces are squares). The result is shown in Fig.7. In this case, there are four classes of spheres leading to four different colours in order that no spheres of the same colour touch each other.

For the case of a cube as the basis for the initial spheres, the angle between the overlapping inversion spheres is also $\frac{\pi}{3}$ as in the case of the tetrahedron, since the conjugate of a cube is an octaderon whose faces are triagular too. So, in this case the inversion algorithm also produces an allowed packing. The result is shown in Fig.8. In this case there are three classes and therefore three colours of spheres.

For the same reason as the case of cube, we obtain a packing for the case of the dodecahedron, since the conjugate of a dodecahedron is an icosahedron with trianglar faces. The result is shown in Fig.9. In this case there are four classes and therefore four colours of spheres.

But, for the case of icosahedron we do not find any allowed packing. Because as is shown in Fig.11, the conjugate of an icosahedron is a dodecahedron with pentagonal faces. This leads to the value $\frac{2 \pi}{5}$ for the angle 
between neighbouring inversion spheres, which does not match any value of $\alpha$ in Eq.(1).

In the previous section we discussed the generalization of having nontouching initial circles. This can also be applied in three dimensions. Similar to two dimensions, reducing the size of the initial spheres we also need to expand the inner inversion sphere (the others are same as before) in order to still be perpendicular to the initial spheres. But, due to the values of $\alpha$, one can see that overlappings of the inversion spheres are at their allowed maximum, except for the case of the octahedron for which $\alpha=\frac{\pi}{4}$. In this case the only value of $\alpha$ which leads to an expanded inner inversion sphere and follows Eq.(1) will be $\frac{\pi}{3}$. Therefore, this is the only packing with nontouching initial spheres which can be constructed.

As an important property of this packing, one can see that only two different colours are enough such that no spheres of same colour touch each other, because the initial spheres don't touch each other and only touch the sphere in the center and this geometry is preserved in all scales, due to the self-similar nature of construction of the packing. We call this packing bichromatic.

\section{FRACTAL DIMENSIONS}

As we mentioned before, the obtained packings have self-similar structures, and, therefore, are fractals. The method we use to calculate the fractal dimensions is the same as the one used in ref.[4]. The number of all spheres in a packing with radii larger than $\epsilon$ follows an asymptotic relation $[7,8]$,

$$
N(\epsilon) \sim \epsilon^{-D}
$$

in which $D$ is the fractal dimension of the packing. Figure 12 shows $N(\epsilon)$ in the logarithmic scales for different packings. One can see that, for smaller $\epsilon$ 's the curves become linear whose slope determines the fractal dimension of the corresponding packing. As shown in the inset of the figure, the slope is calculated by linear fitting to an interval containing five points and shifting this interval towards smaller $\epsilon$ 's. The difference between the fractal dimensions reflects the topological difference of the obtained packings. So, the numerical precision is improved until this difference become evident. The fractal dimension of the packing based on tetrahedron has been calculated in Ref.[4] 
to a high degree of precision. It is approximately 2.474 , which is shown in the figure as the lowest value.

We also calculate the fractal dimensions of two cuts of the bichromatic packing; one through the plane containing four vertices of the octahedron, which also passes the center, and another parallel to this plane but 0.5 off the center. The result is shown in Fig.13. The difference between the fractal dimensions of the cuts shows that the packing is not a homogeneous fractal.

\section{CONCLUTION}

We developed an algorithm for three dimensional self-similar space-filling packings of spheres and constructed four till-now-unknown realizations. These packings are topologically different since they possess different fractal dimensoins. Among them there is a packing which can be represented with only two colours such that no spheres of the same colour touch each other. This is a significant property since it leads to the possibility of rotating spheres with no frustration and therefore to space-filling bearings of spheres, as will be shown in a separate paper (ref.[6]).

\section{ACKNOWLEDGMENT}

We would like to thank R. Peikert for explaining their algorithm to us and giving us the code.

\section{References}

[1] H.J. Herrmann, G. Mantica, and D. Bessis 1990 Space-fillig bearings. Phys. Rev. Lett. 56 3223-6.

[2] G. Oron and H. J. Herrmann, Generalization of space-filling bearings to arbitrary loop size. J. Phys. A:Math. Gen. 33 (2000) 1417-1434.

[3] D.W. Boyd, Can. J. Math. 25 (2), 303-322, 1973.

[4] M. Borkovec, W. de Paris, and R. Peikert, The fractal dimension of the Apollonian sphere packing. Fractals 2 (4), 521-526, 1994. 
[5] B. B. Mandelbrot, The Fractal Geometry of Nature (Freeman, San Francisco, 1982).

[6] R. Mahmoodi Baram, H.J. Herrmann, and N. Rivier, Space fillig bearing in three dimensions. accepted for Physical Review Letters, 2003.

[7] D.W. Boyd, Mathematika 20, 170-174, 1973.

[8] D.W. Boyd, Math. Comp. 39 (159), 249-254, 1982. 


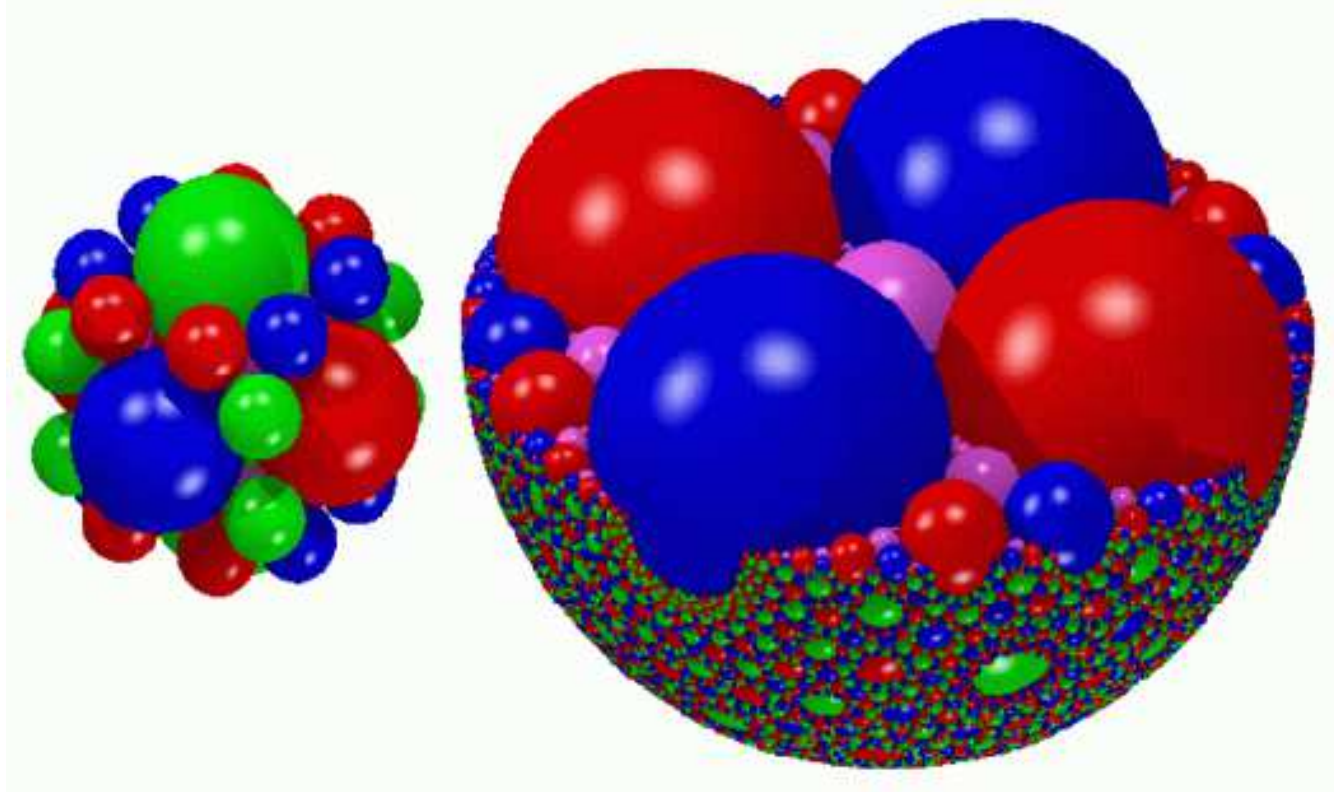

Figure 7: The octahedron-based packing. 


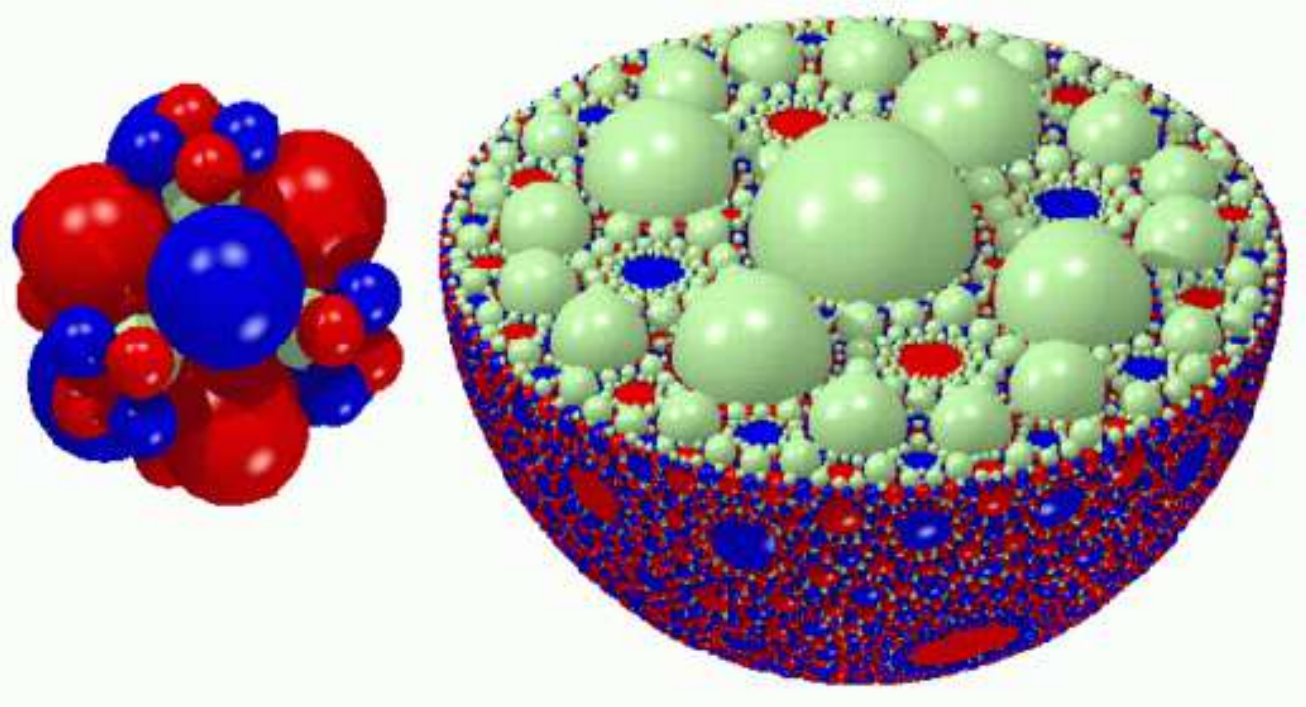

Figure 8: The cube-based packing.

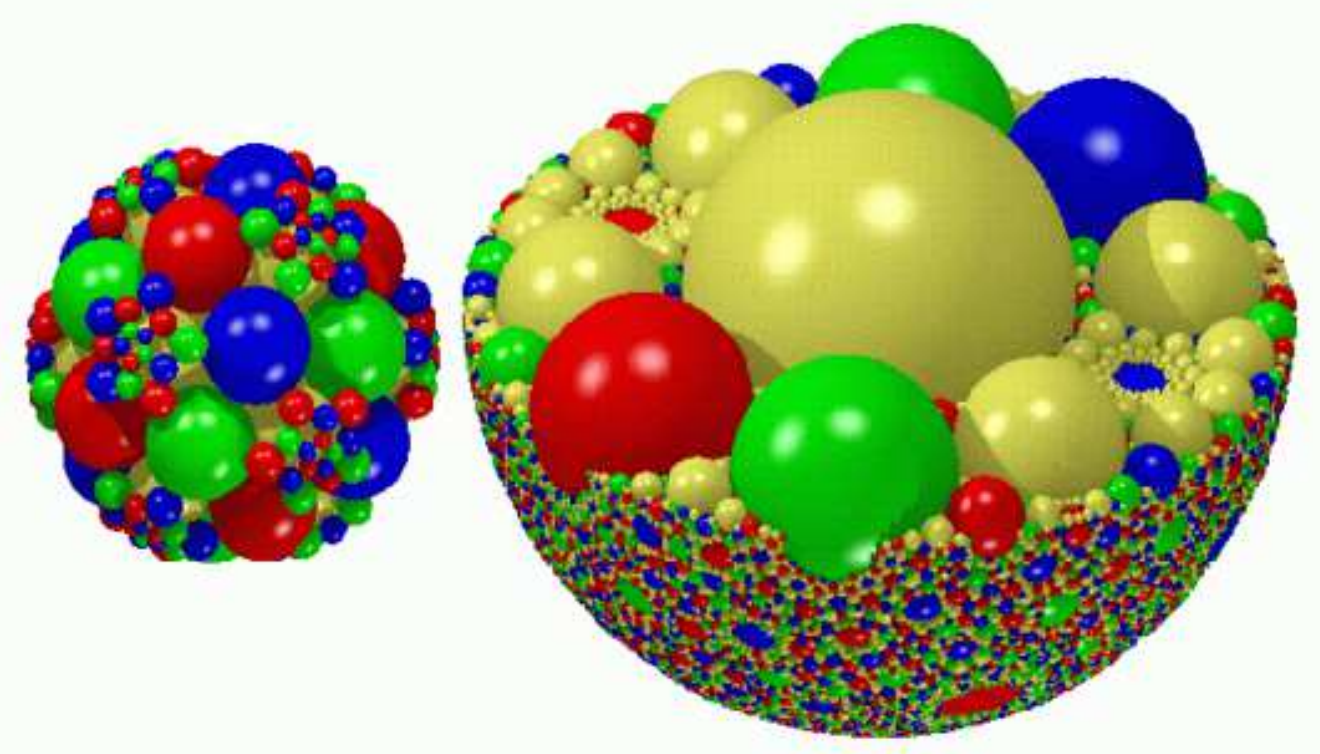

Figure 9: The dodecahedron-based packing. 


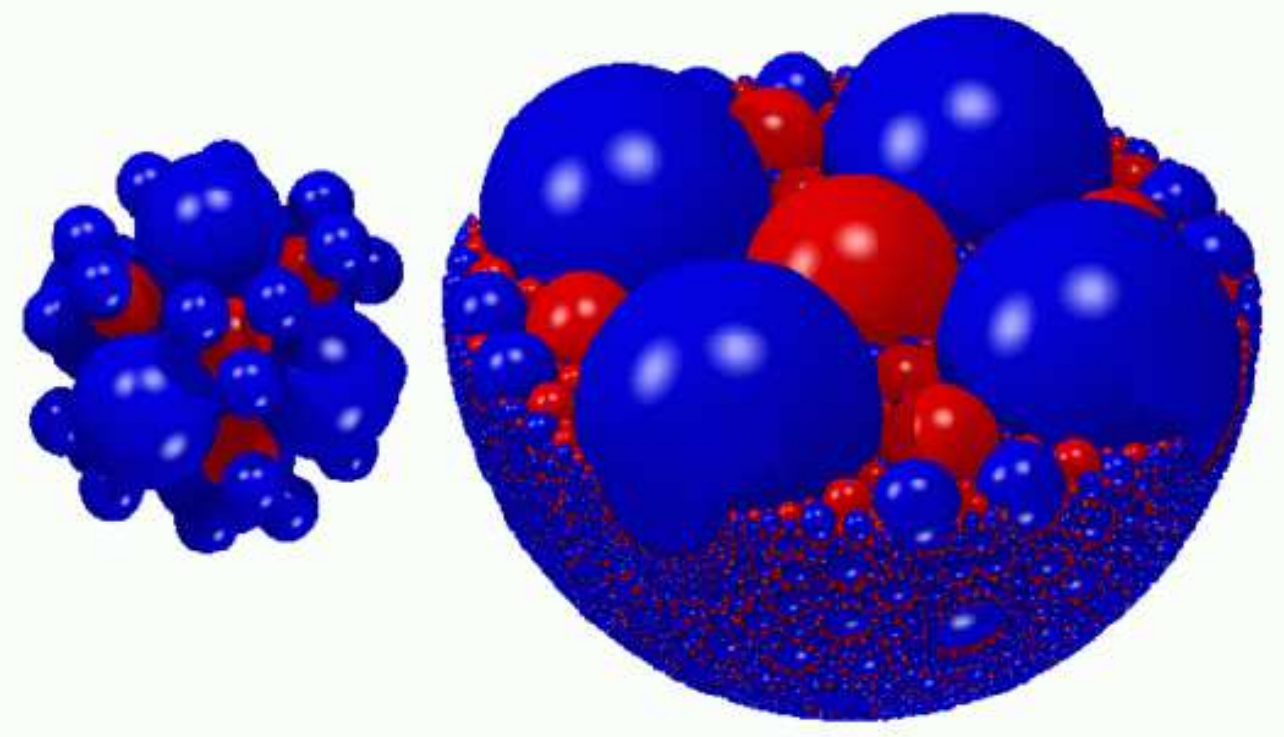

Figure 10: The bichromatic packing which is the second octahedron-based packing. No spheres of the same colour touch each other. 


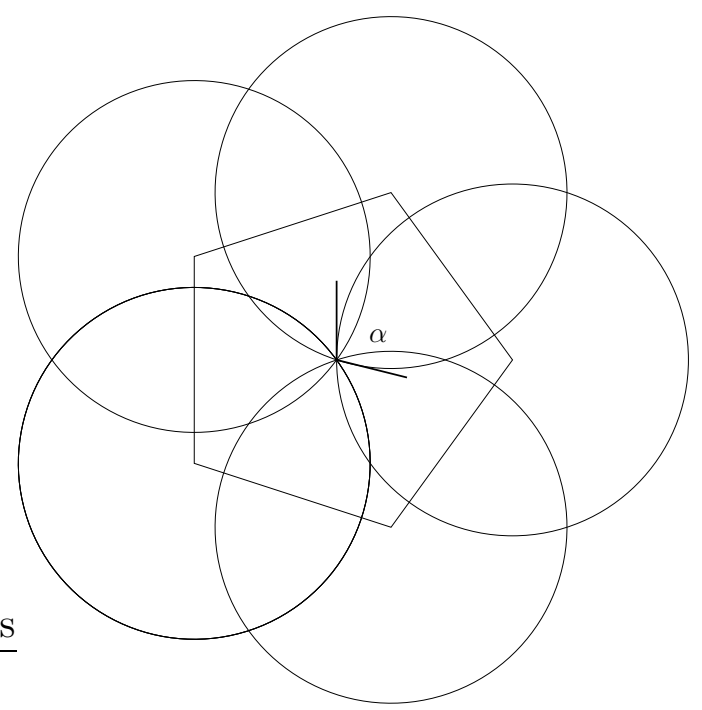

Figure 11: Overlapping of the inversion spheres in the case of the icosahedron. $\alpha$ equals $\frac{2 \pi}{5}$.

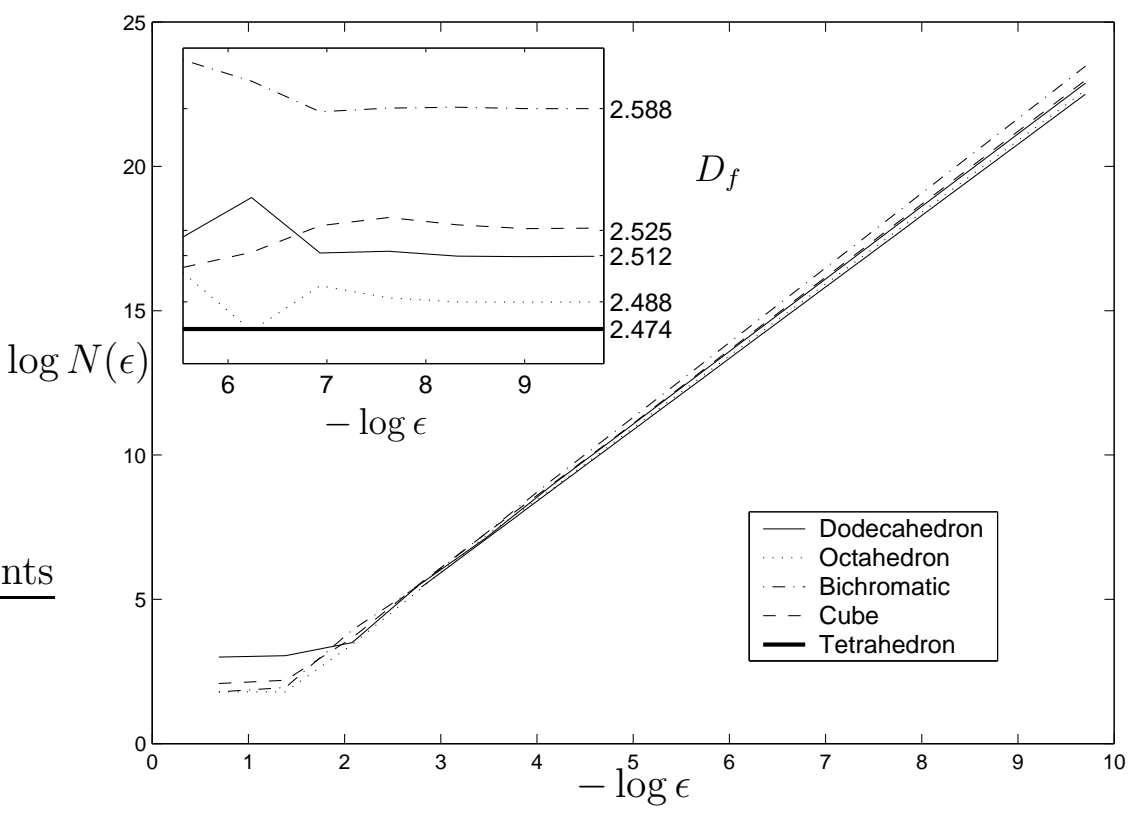

Figure 12: The fractal dimension of different Packings of spheres. 


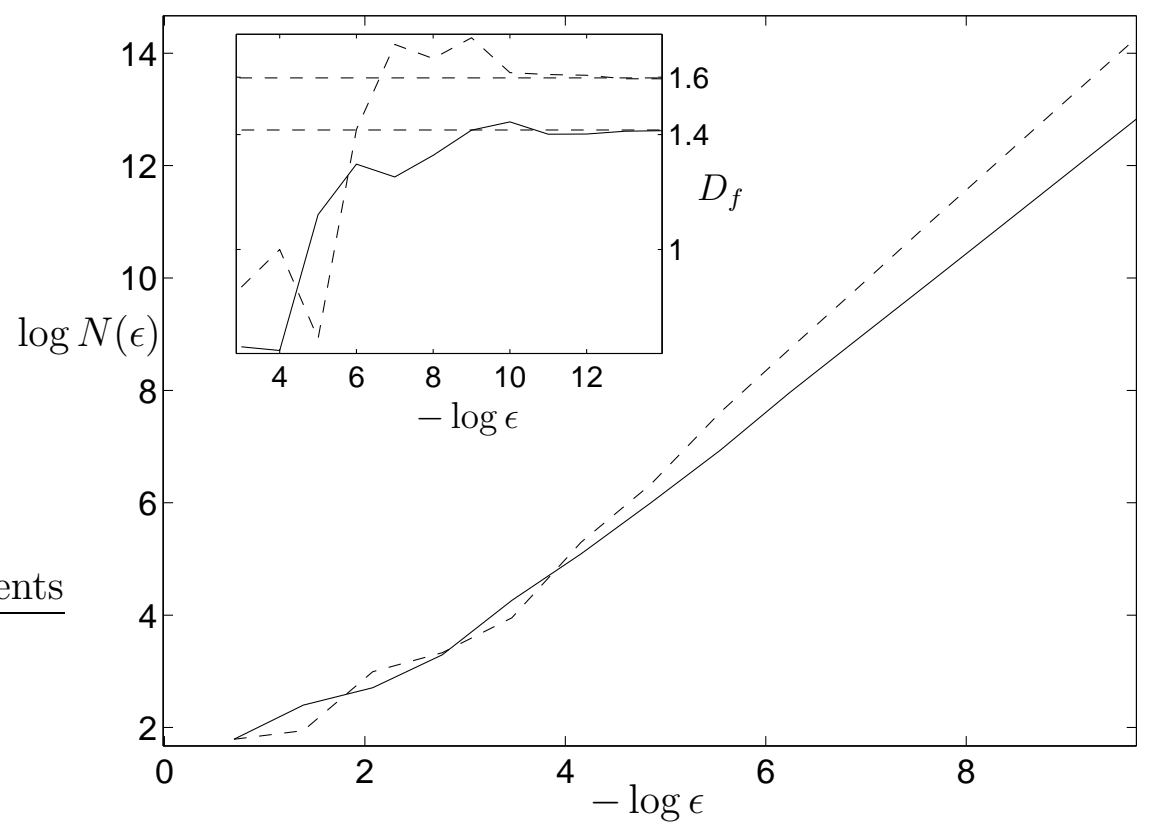

Figure 13: The fractal dimension of two cuts of the bichromatic packing. 